\title{
Organic polymer monolithic columns with incorporated bare and cyano-modified fumed silica nanoparticles for use in hydrophilic interaction liquid chromatography
}

\author{
Nisansala Ganewatta and Ziad El Rassi ${ }^{*}$ (1)
}

\begin{abstract}
This research article presents the preparation and characterization of monolithic columns with incorporated bare fumed silica nanoparticles (FSNPs) and cyano-modified FNSPs (CN-FSNPs) and their subsequent use in hydrophilic interaction liquid chromatography (HILIC) of neutral, polar, and low molecular weight solutes. The monolithic support was based on the in situ polymerization of glyceryl monomethacrylate (GMM) and ethylene glycol dimethacrylate (EDMA) yielding the poly(GMM-CO-EDMA) monolith for the incorporation of bare FNSPs and of CNFSNPs. The poly(GMM-co-EDMA) functioned as a "true support" for bare FSNPs and CN-FSNPs "stationary phases" as manifested by bare FSNPs and CN-FSNPs being the major contributors to solute retention and column selectivity. Overall, the prepared bare FSNPs and CN-FSNPs stationary phases proved useful in HILIC of small polar solutes including dimethylformamide, formamide, thiourea, nucleobases, nucleosides, organic acids, food additives, vitamins, and biological amines.
\end{abstract}

Keywords: High performance liquid chromatography, Hydrophilic interaction liquid chromatography, Organic polymer-based monolith, Fumed silica nanoparticles

\section{Introduction}

Hydrophilic interaction liquid chromatography (HILI C) has gained an increasing attention during the last decade as a promising method for the separation of polar compounds where the use of both reversed phase and normal phase chromatography may be unsuccessful (Jandera, 2011; Jiang et al., 2011). The term "HILIC" was first introduced by Alpert (Alpert, 1990) in 1990, which described this separation mode whereby a relatively nonpolar mobile phase is used to elute the polar analytes that are adsorbed to a moderately polar stationary phase (Gama et al., 2012). The HILIC separation mechanism is multimodal involving intermolecular forces such as electrostatic interactions, hydrogen bonding, dipole-dipole interactions, and weak hydrophobic interactions, all of which may be contributing to solute retention (Gama et al., 2012; Karatapanis et al., 2010). HILIC has been used in the separation of various analytes including peptides, proteins, nucleic acids, carbohydrates, pharmaceutical drugs, and numerous other neutral or charged polar compounds.

\footnotetext{
* Correspondence: ziad.el_rassi@okstate.edu

Department of Chemistry, Oklahoma State University, Stillwater, OK 74078-3071, USA
} 
Silica-based monoliths, which are either bare silica or silica matrix modified with surface-bound polar functional groups, are considered as useful HILIC stationary phases (Buszewski \& Noga, 2012). In addition, organic polymer monoliths (Courtois et al., 2006; Gunasena \& El Rassi, 2013; Holdšvendová et al., 2007) and metal oxidebased stationary phases (Jaoudé et al., 2014; Kučera et al., 2011) have been developed for HILIC separations. Among these numerous stationary phases, monolithic stationary phases are preferred over the particle packed columns due to their unique characteristics such as the ease of preparation and functionalization, high permeability, low resistance to mass transfer, and their stability under extreme $\mathrm{pH}$ conditions (Guiochon, 2007; Svec, 2004). However, the lack of polar monomers and the low solubility of the available monomers in organic porogens have limited the number of useful organic polymer monoliths in HILIC separations. Therefore, post hydrophilization, which involved either the covalent attachment of stratified polar layers (Gunasena \& El Rassi, 2013) or grafting of allyl groups through which polar groups can be attached (Lv et al., 2012), have been successfully implemented to circumvent the abovementioned limitations in the preparation of organic polymer monoliths suitable for HILIC. In addition, Aydoğan and El Rassi (2016a) have recently introduced another interesting approach in obtaining polymer monolithic stationary phases which can be used in HILIC by incorporating fumed silica nanoparticles (FSNPs) as inorganic polar amendment.

Nano-doped stationary phases have more developed surfaces and additional interaction sites provided by the incorporated nano-entities. These nanomaterials also serve as large surface area sorbents and offer different selectivities due to their unique surface adsorption properties (Tong et al., 2013; Y. Xu et al., 2010). Among the various nano-entities which are used in the field of separation sciences, silica nanoparticles (SNPs) have gained some attention due to their distinguished properties such as high surface area, biocompatibility, high organic solvent tolerance, and ease of surface modifications with various functionalities (S. Xu et al., 2017). For instance, SNPs have been used as run buffer additives in separating seven quinolones by capillary zone electrophoresis, in which the analytes were separated within an analysis time of 15 min (Wang et al., 2009).

As has been mentioned above, SNPs can be readily modified with various functionalities via their surface silanol groups. In this regard, amino-modified SNPs were used as additives in capillary electrophoresis for improved enantiomeric separation of four alkaline drugs using carboxymethyl- $\beta$-cyclodextrin as a chiral selector (Gong et al., 2015). In addition, a hybrid monolithic stationary phase with incorporated aminomodified mesoporous silica nanoparticles with surface immobilized pepsin has been recently prepared for the enantioseparation of some basic chiral drugs by capillary electrochromatography (Xu et al., 2017). Furthermore, polymethacrylate-based monolithic column with covalently incorporated modified octadecyl FSNPs have been successfully used in small solutes and protein separations under reversed-phase chromatographic separation conditions (Aydogan \& El Rassi, 2016b). Also, a monolithic stationary phase incorporated with boronic acid-FSNPs also has been prepared for the separation of proteins using nano-liquid chromatography (Aydoğan, 2016).

In this study, FSNPs from Evonik industries, which comes under the trade name of AEROSIL ${ }^{\circ}$ 200, were used. They were manufactured in a continuous flame hydrolysis where $\mathrm{H}_{2}(\mathrm{~g})$ and $\mathrm{O}_{2}(\mathrm{~g})$ are reacted with $\mathrm{SiCl}_{4}$ (l) in a flame at $1000^{\circ} \mathrm{C}$. The $\mathrm{SiO}_{2}$ (s) formation can be described simply as an oxyhydrogen reaction in which water is formed followed by hydrolysis of $\mathrm{SiCl}_{4}$ (l) which yields fumed $\mathrm{SiO}_{2}$ (s) that basically consist of nanoparticles which are referred to as FSNPs. These FSNPs are amorphous, highly dispersed, nonporous, and hydrophilic in nature. They have an average primary particle size of $\sim 12 \mathrm{~nm}$ and $200 \pm 25 \mathrm{~m}^{2} / \mathrm{g}$ specific surface area measured by Brunauer, Emmett, and Teller method using $\mathrm{N}_{2}(\mathrm{~g})$ adsorption and desorption (AEROSIL(R) Fumed silica - technical overview, n.d.).

This paper describes monolithic stationary phases with incorporated FSNPs which were pre-column surface modified with a polar organosilane. Poly(glycerylmonomethacrylate-co-ethylene glycol dimethacrylate) monolithic matrix (referred to as poly(GMM-coEDMA)) was chosen as the ideal support matrix for the incorporation of bare (i.e., unmodified) and surface-modified FSNPs. The prepared monolithic columns were successfully used in the separations of polar compounds under HILIC conditions. The retention factor values, $k$, obtained on these columns were compared with the $k$ values obtained on the monolithic column with entrapped bare FSNPs. To the best of our knowledge, this study demonstrated for the first time, the use of pre-column modified FSNPs incorporated into a polymer monolithic stationary phase to enhance the separation performance of polar molecules under HILIC conditions.

\section{Experimental}

\section{Instruments}

The used HPLC system comprised a pump Model 2350, a gradient programmer Model 2360 and a $\mathrm{V}^{4_{\bullet}}$ variable wavelength absorbance detector from Isco, Inc. (Lincoln, NE, USA), and a Rheodyne injector Model 7010 from 
IDEX Health and Science LLC (Rohnert Park, CA, USA) equipped with a $20 \mu \mathrm{L}$ loop. The chromatograms were occasionally recorded with a Chromatopac CR601 integrator from Shimadzu (Kyoto, Japan). Otherwise, data collection was performed using the PowerChrom software version 2.5.4 from eDAQ (Denistine East, NSW, Australia). The off HPLC system processing of chromatograms was carried out by an OriginPro v8.5.1 from Origin Lab Corp., (Northampton, MA, USA). The in situ polymerization of monolithic HPLC columns was carried out in a water bath equipped with an immersion circulator Model Isotemp 2100 from Fisher Scientific (Waltham, MA, USA). After the polymerization, the porogens and the unreacted materials were washed off using a Waters Model 590 HPLC pump from Waters division of Millipore (Milford, MA, USA). The ultrasonic cleaner model 1510R-MTH from Branson Ultrasonic Corporation (Danbury, CT, USA) was used for low power sonication. Model Centra CL3R centrifuge machine from International Equipment Company (Needham Heights, MA, USA) was used in isolating the surface-modified FSNPs from its reaction solvent medium by centrifugation. In characterizing the surface modified FSNPs, all the Fourier transform infrared (FTIR) analyses were carried out at the attenuated total reflectance mode using a Nicolet IS50 FT-IR machine from Thermo Scientific company (Waltham, MA, USA). In addition, the Q-50 thermogravimetric analyzer from TA instruments (New Castle, DE, USA) was used to perform the thermogravimetric analyses (TGA) in which surface-modified FSNPs were heated from 25 to $750{ }^{\circ} \mathrm{C}$ at a heating rate of $20{ }^{\circ} \mathrm{C} / \mathrm{min}$ under a $40 \mathrm{~mL} / \mathrm{min}$ of continuous $\mathrm{N}_{2}$ (g) flow.

\section{Reagents and materials}

AEROSIL 200 FSNPs were gifted from Evonik Corporation (Parsippany, NJ, USA). Glyceryl monomethacrylate (GMM) was purchased from Monomer Polymer and Dajac Labs (Trevose, PA, USA). Ethylene glycol dimethacrylate (EDMA), cyclohexanol, 1dodecanol, thiourea, uracil, adenine, cytosine, uridine, adenosine, cytidine, 1-phenylheptane, thiamine $\mathrm{HCl}$, $( \pm)$-phenylpropanolamine $\mathrm{HCl},( \pm)$-propranolol $\mathrm{HCl}$, and $( \pm$ )-ephedrine $\mathrm{HCl}$ were purchased from Sigma Chemical Co. (St. Louis, MO, USA). 2,2'-Azobis(isobutyronitrile) (AIBN) and L-ascorbic acid were obtained from Aldrich Chemical Co. (Milwaukee, WI, USA). Thymine was from the Nutritional Biochemical Cooperation (Cleveland, OH, USA). N,N-Dimethylformamide (DMF) and formamide were obtained from EM Science (Gibbstown, NJ, USA). ACS grade acetonitrile (ACN), isopropanol (IPA), and glacial acetic acid were purchased from Pharmco-AAPER (Brookfield, CT, USA). $m$-Toluic acid, maleic acid, and riboflavin were from Eastman Organic Chemicals (Rochester, NY, USA). Salicylic acid and benzoic acid were obtained from J. T. Baker Chemical Co., (Phillipsburg, NJ, USA). Sulfanilic acid was from American Tokyo Kasei, Inc., (Portland, OR, USA). Sulfosalicylic acid and $4 \AA$ effective pore size molecular sieves were purchased from Fisher Scientific Company (Fairlawn, NJ, USA). Nicotinamide was from Alfa Aesar (Haysham, Lancaster). Ammonium acetate and ACS reagent grade formic acid were obtained from Spectrum Quality Products Inc., (Gardena, CA, USA). 2Cyanoethyltrichlorosilane was from Hüls Petrarch Systems (Bristol, PA, USA). All the HPLC column end fittings were obtained from Crawford Fitting Co., (Solon, OH, USA). Stainless steel tubing with $4.6 \mathrm{~mm}$ i.d. was purchased from Alltech Associates (Deerfield, IL, USA) and was cut in house to the desired column lengths.

\section{Preparation of poly(GMM-co-EDMA) monolithic HPLC column incorporated with bare FSNPs}

Due to the nano size of the bare and modified FSNPs, it is essential to support them on a good support medium with favorable flow characteristics. In this regard, poly(GMM-co-EDMA) monolithic support was considered as the ideal matrix for the incorporation of bare and surface-modified FSNPs, which was originally developed and optimized by Mayadunne and El Rassi (2014). This basic monolithic support is presented in Fig. 1a that shows the process involved in the in situ polymerization of the functional monomer GMM and the crosslinker EDMA in the presence of a binary porogenic solvent consisting of cyclohexanol and 1-dodecanol. The GMM provides a hydrophilic diol surface to the poly(GMM-co-EDMA) monolith which imparts hydrophilic interactions while EDMA introduces some non-polar character to the surface.

The monolithic column with physically entrapped FSNPs was directly synthesized by in situ polymerization in a stainless-steel column tube. With minimum adjustments in the ratios of the monomers and porogens, the preparation method was basically similar to the initial work described by Aydoğan and El Rassi (2016a), who introduced the FSNPincorporated hydrophilic monolithic columns for HILI C separations for the first time. In preparing the polymerization mixture which had a final weight of $2.88 \mathrm{~g}, 29.59 \mathrm{wt} \%$ of GMM, $13.31 \mathrm{wt} \%$ of EDMA, and $0.35 \mathrm{wt} \%$ of FSNPs were added to the binary porogenic mixture that consisted of $30.23 \mathrm{wt} \%$ of cyclohexanol and $26.25 \mathrm{wt} \%$ of 1-dodecanol. Then, 0.27 wt $\%$ of AIBN was added, and the mixture was vortexed for $2 \mathrm{~min}$ followed by low power bath 
A

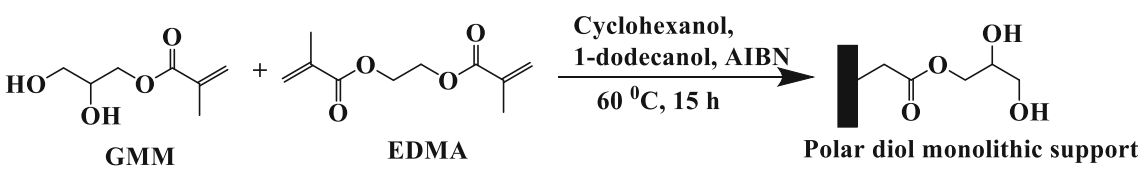

B

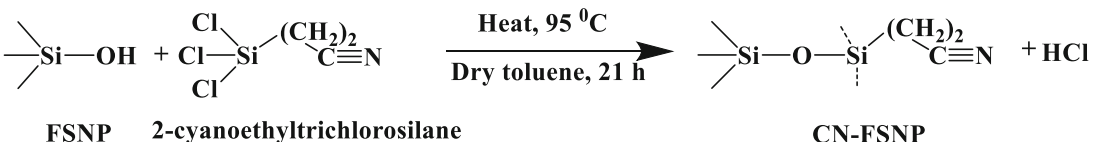

Fig. 1 Schematics of the fabrication of poly(GMM-Co-EDMA) monolithic support (a) and preparation of CN-FSNPs (b)

sonication for $20 \mathrm{~min}$. The homogenous polymer solution thus obtained was introduced into a two section $15-\mathrm{cm}$ long column comprising a $10 \mathrm{~cm} \times 4.6 \mathrm{~mm}$ i.d. section connected to a $5 \mathrm{~cm} \times 4.6 \mathrm{~mm}$ i.d. section through a $1 / 4$ in. union. Here, the additional $5-\mathrm{cm}$ long column was used to make sure that the $10-\mathrm{cm}$ long analytical column will be fully formed without voids or contractions. Thereafter, both column ends were plugged tightly, and the column was kept in a water bath at $60{ }^{\circ} \mathrm{C}$ for $15 \mathrm{~h}$. After the completion of the polymerization period, the monolithic column was washed with $80 \%(\mathrm{v} / \mathrm{v})$ ACN using Waters HPLC pump, in order to remove the porogens and the unreacted materials. Next, the top $5-\mathrm{cm}$ column was detached from the $10-\mathrm{cm}$ separation column which was then equilibrated with the mobile phase for $1 \mathrm{~h}$ prior to chromatographic analyses. These FSNPentrapped monolithic columns are referred to as FSNPs-poly(GMM-co-EDMA) in the current study.

\section{Preparation of FSNPs with cyano surface functionalities}

Typically, $0.1 \mathrm{~g}$ of FSNPs was suspended in $10 \mathrm{~mL}$ of toluene (pre-dried using molecular sieves of $4 \AA$ effective pore size) in a round-bottomed flask connected to a reflux condenser. At the beginning of the reaction, this mixture was heated to $95{ }^{\circ} \mathrm{C}$ with slow magnetic stirring for $30 \mathrm{~min}$. Thereafter, $0.1 \mathrm{~g}$ of 2 cyanoethyltrichlorosilane was added to this suspension, and the resulting mixture was stirred for $21 \mathrm{~h}$ at $95{ }^{\circ} \mathrm{C}$. Next, the reaction mixture was cooled down to room temperature and centrifuged at $4000 \mathrm{rpm}$ for $20 \mathrm{~min}$ to isolate the resulting surface-modified FSNPs with $\mathrm{CN}$ groups and referred to as CN-FSNPs. The isolated CN-FSNPs were rinsed with acetone and were allowed to dry in the air. This surface modification reaction is depicted in Fig. 1b.
Preparation of poly(GMM-co-EDMA) monolithic HPLC columns with incorporated CN-FSNPs

The CN-FSNP-entrapped monolithic columns which are referred to as CN-FSNPs-poly(GMM-co-EDMA) were prepared via direct in situ polymerization within a $15-\mathrm{cm}$ long stainless-steel column. Here, in preparing the polymerization solution which had a final weight of $2.88 \mathrm{~g}, \mathrm{GMM}$ at $29.59 \mathrm{wt} \%$, EDMA at 13.31 $w \mathrm{t} \%$, and CN-FSNPs at varying wt $\%$ in the $0.35-2.77$ $\mathrm{wt} \%$ range were added to the binary porogenic solvent mixture consisting of cyclohexanol at 29.01-30.23 $\mathrm{wt} \%$ and 1-dodecanol at 25.03-26.25 wt\% in order to complete to a total of $100 \mathrm{wt} \%$ with that of the wt\% of CN-FSNPs. Next, AIBN 0.27 wt\% was added, and the resulting polymerization solution was vortexed for $2 \mathrm{~min}$ followed by low power sonication for $20 \mathrm{~min}$ before introducing it into the $15-\mathrm{cm}$ long stainlesssteel column which was assembled and used as described in the preparation of bare FSNPs incorporated monolithic columns. Thereafter, both column ends were tightly plugged, and the column was heated at $60{ }^{\circ} \mathrm{C}$ for $15 \mathrm{~h}$ to allow the polymerization. Then, the resulting monolithic column was washed with $80 \%$ (v/ $\mathrm{v}) \mathrm{ACN}$ to remove the porogens and the unreacted materials. After removing the extra $5 \mathrm{~cm}$ column section, the $10-\mathrm{cm}$ long separation column was equilibrated with the desired mobile phase prior to chromatographic evaluation.

\section{Chromatographic conditions for HILIC separations and evaluation of solute retention from the chromatogram} The column effluent was monitored at wavelengths of $214 \mathrm{~nm}$ and $254 \mathrm{~nm}$ while pumping at a flow rate of $1 \mathrm{~mL} / \mathrm{min}$ at room temperature. Solution mixtures containing $\mathrm{ACN}$ and either ammonium acetate or water were used as the mobile phase. The $\mathrm{pH}$ of the mobile phase is reported with respect to the aqueous 
portion. Formic acid or ammonium hydroxide was used in adjusting the $\mathrm{pH}$ of the aqueous ammonium acetate before mixing it with ACN. All the mobile phases were freshly prepared, and low power bath sonication for $1 \mathrm{~h}$ was used to degas the eluent before use. The retention factors, $k$, were determined as follows

$$
k=\frac{t_{R}-t_{0}}{t_{0}}
$$

where $t_{0}$ is the retention time of the dead time marker (toluene or phenyl heptane) and $t_{\mathrm{R}}$ is the solute retention time.

\section{Results and discussion}

Evaluation of the mechanical and chromatographic properties of FSNPs-poly(GMM-co-EDMA) monolith

The mechanical stability of the monolithic column which was prepared by incorporating $0.35 \mathrm{wt} \%$ FSNPs into the polymerization solution (i.e., $0.6 \mathrm{mg} F S N P s / \mathrm{cm}$ ) was evaluated by monitoring the column backpressure at varied flow rates of the mobile phase. In this regard, a linear relationship $\left(y=1090.5 \times-885.95\right.$ and $R^{2}=$ 0.991 ) was obtained within the tested range of flow rate $(0.8-1.4 \mathrm{~mL} / \mathrm{min})$ and the range of resulting backpressure (20-660 psi) when using $\mathrm{ACN}$ as the mobile phase. This is a clear indication of the good mechanical stability of the prepared FSNPs-poly(GMM-coEDMA) monolith.

HILIC analyses are generally performed using organicrich mobile phases. Therefore, all the chromatographic analyses were carried out using either $90 \%(\mathrm{v} / \mathrm{v})$ or $95 \%$ $(\mathrm{v} / \mathrm{v})$ ACN content in the mobile phase. The remainder of the mobile phase was either water or aqueous ammonium acetate solution. The retention characteristics of the prepared FSNPs-poly(GMM-co-EDMA) monolith was evaluated using a wide range of polar solutes including DMF, formamide, thiourea, nucleobases, organic acids, food additives, vitamins, and biological amines. All the analyses were performed under isocratic elution conditions.

Small polar neutral solutes A neutral polar solute mixture consisting of DMF, formamide, and thiourea was baseline separated on the prepared FSNPs-poly(GMMco-EDMA) monolithic column as can be seen in Fig. 2a. These tested solutes were eluted in the order of increasing polarity showing the typical HILIC behavior where the least polar DMF eluted first while the most polar thiourea, which is usually used as the dead time marker in reversed phase chromatography, eluted last. The
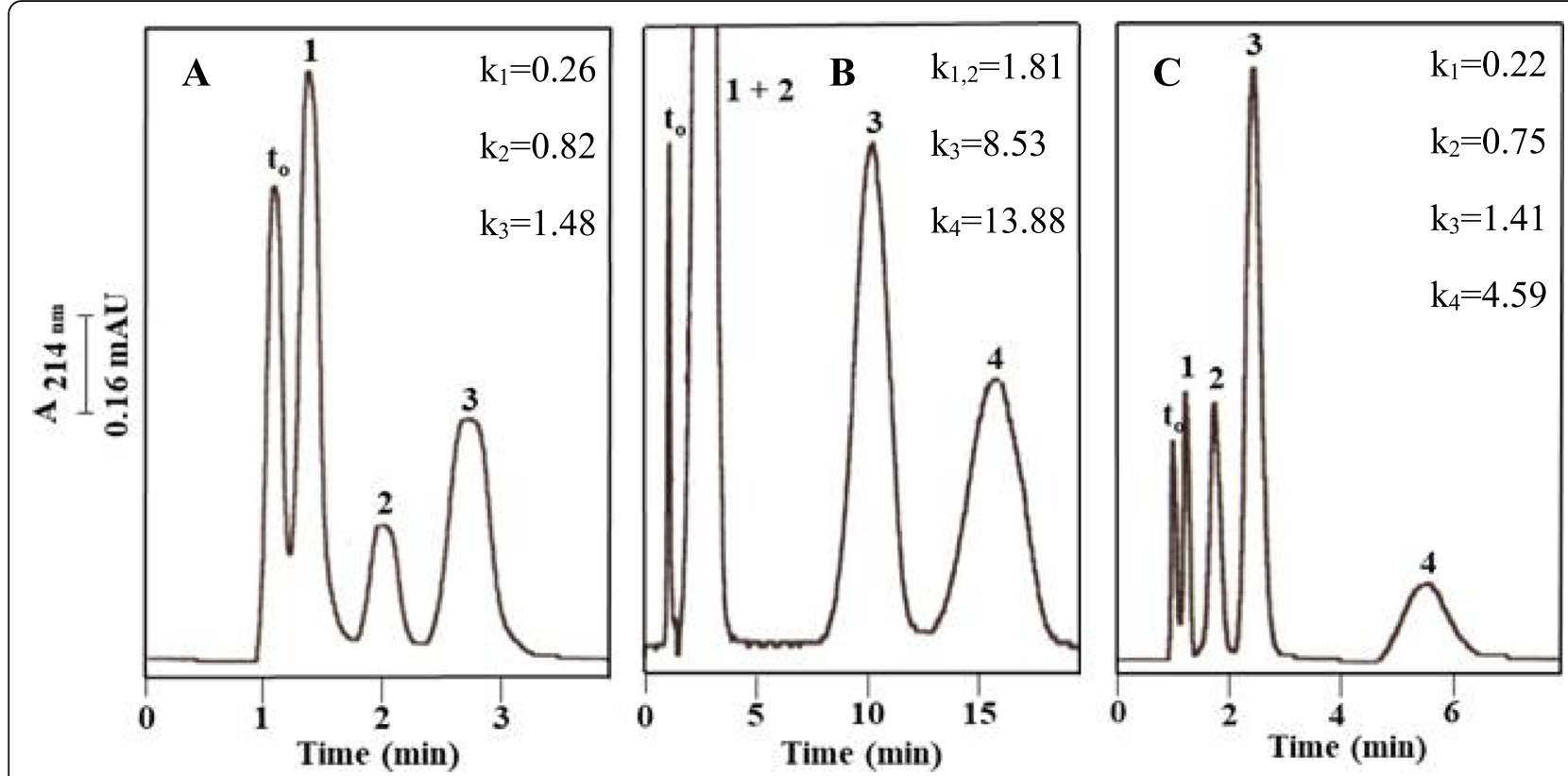

Fig. 2 Chromatograms of small neutral polar solutes (a), nucleobases (b), and organic acids (c) obtained on FSNPs-poly(GMM-co-EDMA) monolithic column. Separation conditions: column, $10 \mathrm{~cm} \times 4.6 \mathrm{~mm}$ i.d with $0.6 \mathrm{mg}$ of FSNPs/cm; flow rate, $1 \mathrm{~mL} / \mathrm{min}$; injection volume, 20 HL; column temperature, room temperature. For a, mobile phase, 95\% ACN (v/v), 5\% water (v/v); solutes: 1, DMF; 2, formamide; 3, thiourea; $\mathrm{t}_{0}$, toluene. For b, mobile phase, 95\% ACN (v/v), 5\% of $10 \mathrm{mM}$ ammonium acetate, pH 4.0 (v/v); solutes: 1, thymine; 2, uracil; 3, adenine; 4, cytosine; $\mathrm{t}_{0}$, toluene. For c, mobile phase, $95 \%$ ACN (v/v), 5\% of $10 \mathrm{mM}$ ammonium acetate, pH 4.0 (v/v); solutes: 1, m-toluic acid; 2 , salicylic acid; 3, sulfanilic acid; 4, sulfosalicylic acid; $t_{0}$, toluene 
average separation efficiency was only 2700 plates $/ \mathrm{m}$. However, the values of the selectivity factors, $\alpha$, were noticeably as high as 3.2 and 1.8 for the solute pairs $2 / 1$ and $3 / 2$, respectively, ensuring a good separation for the solutes.

Nucleobases The FSNPs-poly(GMM-co-EDMA) monolith was further characterized using a mixture of 4 nucleobases including thymine, uracil, adenine, and cytosine, as shown in Fig. 2b. The nucleobase test solutes were eluted in the order of increasing polarity confirming the usual HILIC separation behavior. The $\alpha$ values were significantly high with numerical values of 4.7 and 1.6 for the solute pairs $3 / 1,2$ and $4 / 3$, respectively.

Organic acids A mixture of 4 organic acids including $m$-toluic acid, salicylic acid, sulfanilic acid, and sulfosalicylic acid was analyzed on the prepared FSNPspoly(GMM-co-EDMA) monolithic column. These tested organic acids were eluted showing the typical HILIC chromatographic elution behavior where the most polar sulfosalicylic acid with polar functionalities was eluted last while the least polar $m$-toluic acid eluted first as can be seen in Fig. 2c. The $\alpha$ values were 3.5, 1.9, and 3.3 for the solute pairs $2 / 1,3 / 2$, and $4 / 3$, respectively.

Food additives Food additives are widely used in order to preserve the quality and to enhance the flavor, color, and the texture of the foods. In this investigation, some of the commonly used acid type food additives including benzoic acid (i.e., E210), maleic acid (E296), and ascorbic acid (E300) were separated isocratically under HILIC conditions on the FSNPs-poly(GMM-co-EDMA) monolithic column. The $\alpha$ values were 1.7 and 6.4 for the solute pairs $2 / 1$ and $3 / 2$, respectively. The obtained chromatogram is shown in the Fig. 3a.

Vitamins Vitamins are organic compounds which are essential in minute amounts for the body's physiological functions. Inadequate amounts of vitamins in the diet often results in vitamin deficiency related diseases (Combs Jr \& McClung, 2016). The FSNPs-poly(GMMco-EDMA) monolithic column was further tested in the separation of vitamins under HILIC conditions. In this regard, nicotinamide $\left(\mathrm{pK}_{\mathrm{a}}=3.35\right)$, riboflavin (i.e., vitamin $\mathrm{B}_{2}, \mathrm{pK}_{\mathrm{a}}=10.2$ ), and thiamine (i.e., vitamin $\mathrm{B}_{1}, \mathrm{pK}_{\mathrm{a}}=4.8$ ) were chromatographed on the monolithic column under investigation with $\alpha$ values of 2.8 and 6.6 for the solute pairs of $2 / 1$ and $3 / 2$, respectively. The obtained chromatogram is displayed in Fig. 3b.

Biological amines The separation ability of the FSNPspoly(GMM-co-EDMA) monolithic column under investigation was further evaluated using 3 biological amines including $( \pm)$-phenylpropanolamine, $( \pm)$-propranolol, and $( \pm)$-ephedrine as shown in Fig. 3c. The obtained $\alpha$ values were as high as 3.6 and 2.1 for the solute pairs of

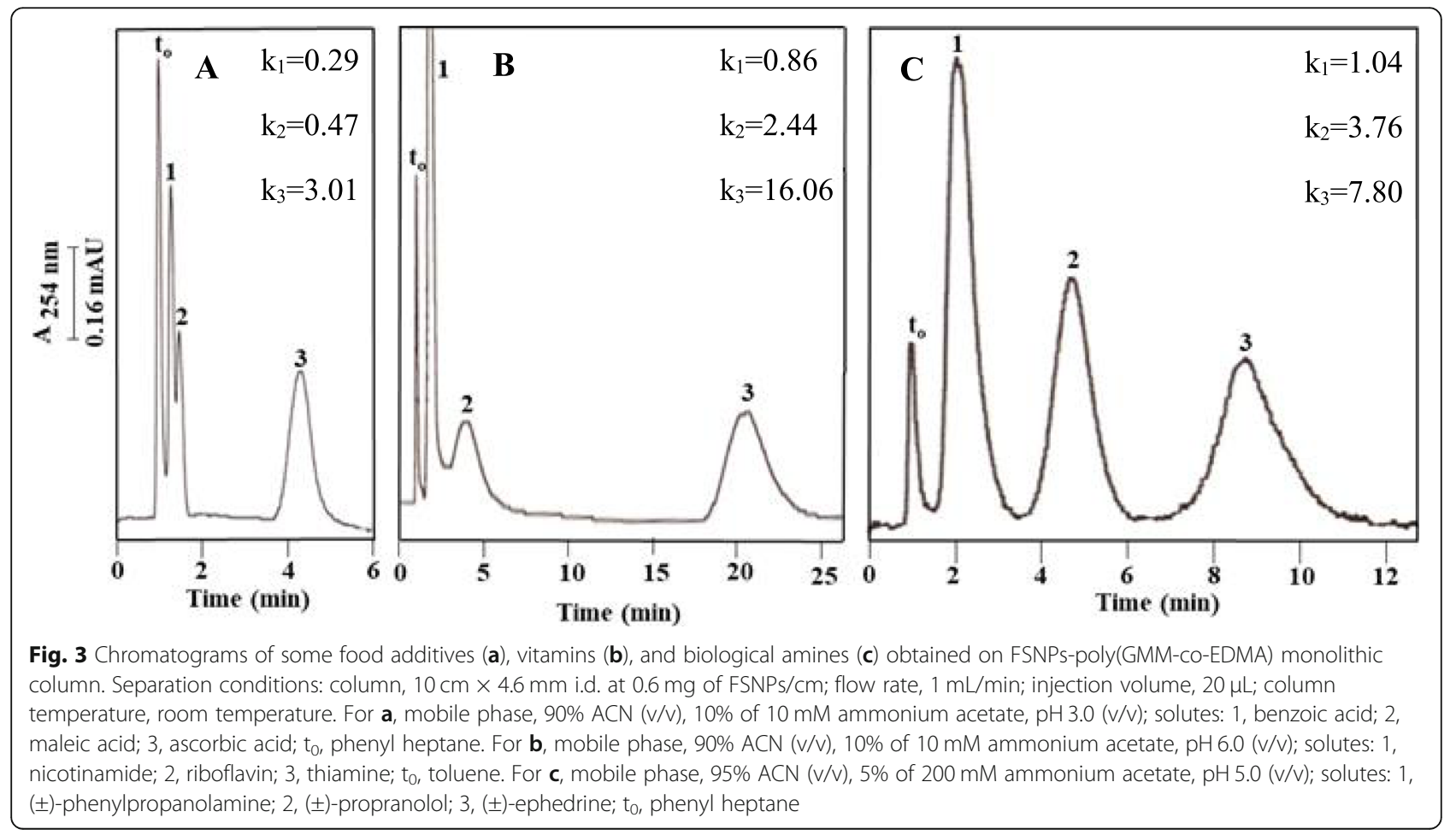


$2 / 1$ and $3 / 2$, respectively, which permitted their baseline separations even though the peaks look relatively broad.

Reproducibility The reproducibility of the prepared monolithic stationary phase containing $0.6 \mathrm{mg}$ of incorporated FSNPs per $\mathrm{cm}$ column length was evaluated using small neutral polar solutes including DMF, formamide, and thiourea as models. This was carried out by measuring their $k$ values from run-to-run $(n=3)$, dayto-day $(n=3)$, and column-to-column $(n=2)$ using a mobile phase at $95 \%(\mathrm{v} / \mathrm{v}) \mathrm{ACN}$ and $5 \%(\mathrm{v} / \mathrm{v})$ water. The day-to-day and column-to-column reproducibilities were calculated by averaging triplicate measurements of the $k$ values on each day and on each column. The average \%RSDs were $2.79 \%, 2.83 \%$, and $2.49 \%$ for run-to-run, day-to-day, and column-to-column, respectively.

\section{Monolithic columns with incorporated cyano-modified FSNPs}

Characterization of cyano-modified FSNPs The silanol groups present on the FSNPs allow the convenient introduction of various functional groups of interests onto its surface by reacting them with organosilanes. In this regard, the FSNPs were allowed to react with a polar organosilane in order to obtain surface-modified FSNPs with polar functionalities which were then incorporated into the given monolithic columns for HILIC separations. Particularly, the FSNPs were reacted with 2cyanoethyltrichlorosilane to obtain cyano-modified FSNPs referred to as CN-FSNPs as shown in Fig. $1 \mathrm{~b}$.

The bonding of cyano functionalities to FSNPs was verified by FTIR and TGA. The FTIR spectra obtained for bare and CN-FSNPs in the range of 500 to 4000 $\mathrm{cm}^{-1}$ are shown in Fig. 4. The prominent band in the region of $1130-1000 \mathrm{~cm}^{-1}$ which can be seen in both spectra corresponds to siloxane $(\mathrm{Si}-\mathrm{O}-\mathrm{Si})$ stretching. In addition, the single broad band which is in the range of $950-810 \mathrm{~cm}^{-1}$ resembles $\mathrm{Si}-\mathrm{OH}$ stretching. Furthermore, CN-FSNPs spectrum shows characteristic bands in the ranges of $2850-3000 \mathrm{~cm}^{-1}, 1350-1470 \mathrm{~cm}^{-1}$, and $720-725 \mathrm{~cm}^{-1}$ arising from stretching, bending, and rocking of $\mathrm{CH}_{2}$ groups, respectively. The less intense band in the range of $2240-2260 \mathrm{~cm}^{-1}$ is due to the stretching vibrations of the nitrile group. These characteristic bands on the CN-FSNPs confirm the covalent attachment of the desired polar organosilane onto the surface of the FSNPs.

The thermogravimetric analyses were also performed on CN-FSNPs and FSNPs by heating them from 25 to $750{ }^{\circ} \mathrm{C}$. A sample of $0.7460 \mathrm{mg}$ was used for the TG analysis. The TG curve thus obtained is shown in Fig. 5. Based on this thermogram, CN-FSNPs showed three distinct thermal events in the range of $120-750{ }^{\circ} \mathrm{C}$ which is

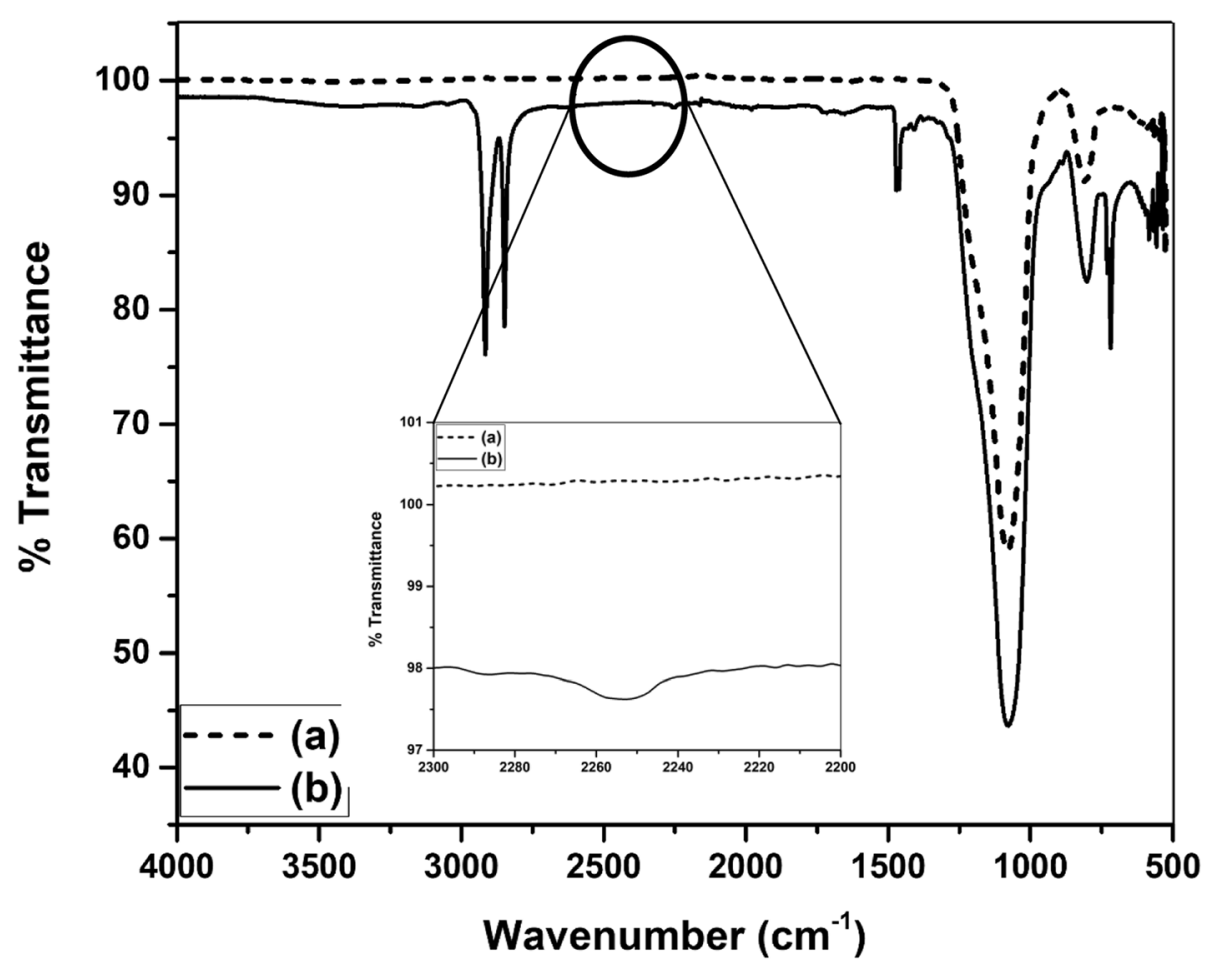

Fig. 4 FTIR spectra of FSNPs (a) and CN-FSNPs (b) 


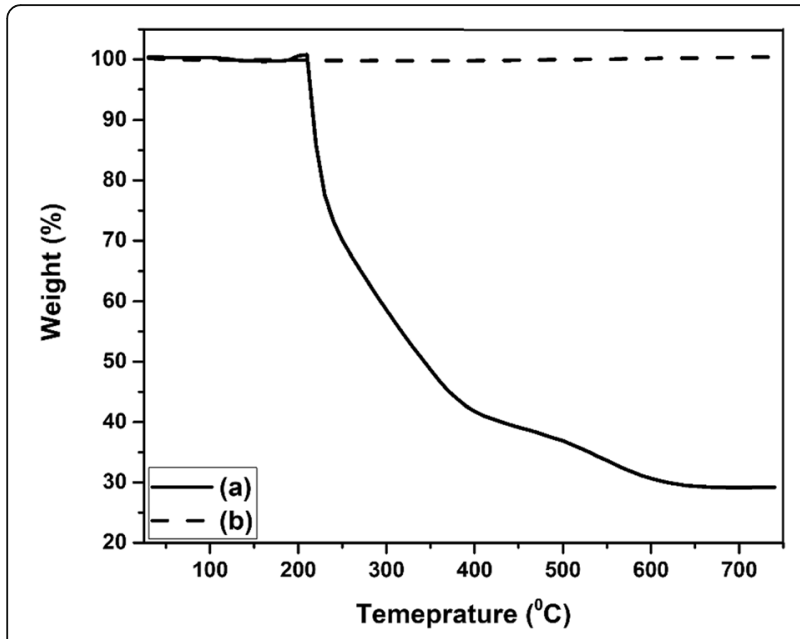

Fig. 5 TG curve of CN-FSNPs (a) and bare FSNPs (b)

attributed to the thermal decomposition of silica surface bonded organosilane moieties while the weight of bare FSNPs remained constant. Moreover, the corresponding $70.46 \%$ weight loss (see Fig. 9) reveals a surface coverage of $144 \mu \mathrm{mol}$ of ligands $/ \mathrm{m}^{2}$ from the surface area of the FSNPs. These results further confirm the successful attachment of the desired polar organosilane onto the surface of the FSNPs. Given the fact that only half of the $8 \mu \mathrm{mol} / \mathrm{m}^{2}$ silanols could be reacted with organosilanes due to steric hindrance (Melander et al., 1980), the $144 \mu \mathrm{mol} / \mathrm{m}^{2}$ ligands indicate the presence of multilayer coating with 2-cyanoethyltrichlorosilane. This is not surprising because trichlorosilanes in general are known to yield polymeric coating on silica surface (Melander et al., 1980).

Evaluation of chromatographic and mechanical properties of CN-FSNPs-poly(GMM-co-EDMA) monolithic column

The effect of the covalent attachment of 2cyanoethyltrichlorosilane to the FSNPs' surface was evaluated by comparing the $k$ values of the tested polar analytes obtained on the CN-FSNPs-poly(GMM-co-EDMA) column with those obtained on FSNPs-poly(GMM-coEDMA) column, each contained $0.6 \mathrm{mg}$ of FSNPs or $\mathrm{CN}$-FSNPs per $\mathrm{cm}$ column length. In order to find the optimum amount of CN-FSNPs that can be incorporated into the monolithic column and to investigate the effect of the CN-FSNPs content in the monolithic column on solute retention, a series of monolithic columns was prepared by varying the wt\% $(0.35-2.8 \mathrm{wt} \%)$ of $\mathrm{CN}$ FSNPs in the polymerization solution. The separation efficiencies of these prepared monolithic columns with various amounts of $\mathrm{CN}$-FSNPs were evaluated using a set of neutral, low molecular weight, and polar solutes including DMF, formamide, and thiourea as model test solutes. The obtained average separation efficiencies of these solutes on the prepared series of columns are shown in Fig. 6. As can be seen in this figure, the average separation efficiencies of the tested solutes decreased with increasing the amount of incorporated CN-FSNPs into the monolithic column. This decrease in the separation efficiency might have arisen from the adverse effects of incorporating large amounts of CN-FSNPs in the monolithic structure formation. Therefore, $0.35 \mathrm{wt} \%$ of CN-FSNPs was identified as the optimum amount of $\mathrm{CN}-\mathrm{FSNPs}$ that can be incorporated into the polymerization solution based on the highest separation efficiency achieved on the $0.6 \mathrm{mg}$ of CN-FSNPspoly(GMM-co-EDMA) column which was prepared from a polymerization solution with $0.35 \mathrm{wt} \% \mathrm{CN}$ FSNPs.

The backpressure measurements obtained on the monolithic columns with various amounts of incorporated CN-FSNPs showed that the structure of the monolith strongly depended upon the CN-FSNPs content in the polymerization solution. When using $80 \% \mathrm{ACN}(\mathrm{v} / \mathrm{v})$ and $20 \%$ water $(\mathrm{v} / \mathrm{v})$ as the mobile phase, linear relationships $\left(R^{2}>0.99\right)$ between the flow rate and the resulting backpressure were obtained for all the prepared monolithic columns thus indicating a good mechanical stability of CN-FSNPs-(poly GMM-co-EDMA) monolithic columns. Moreover, the obtained column backpressure

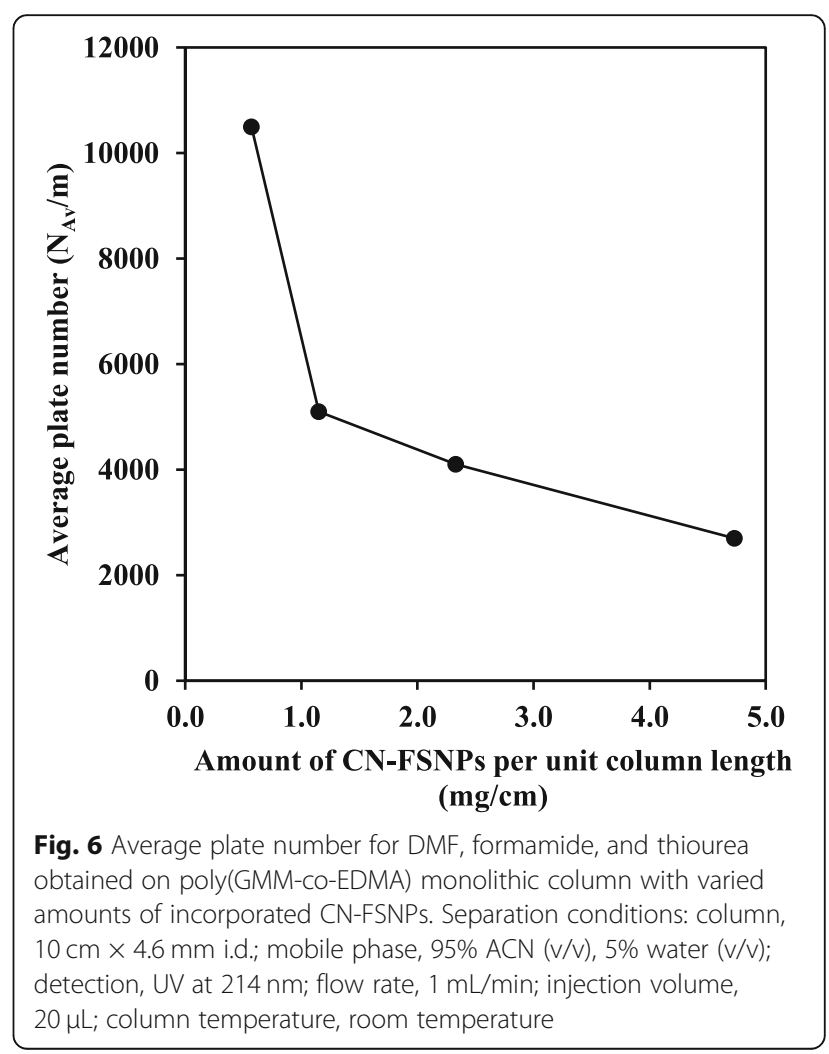



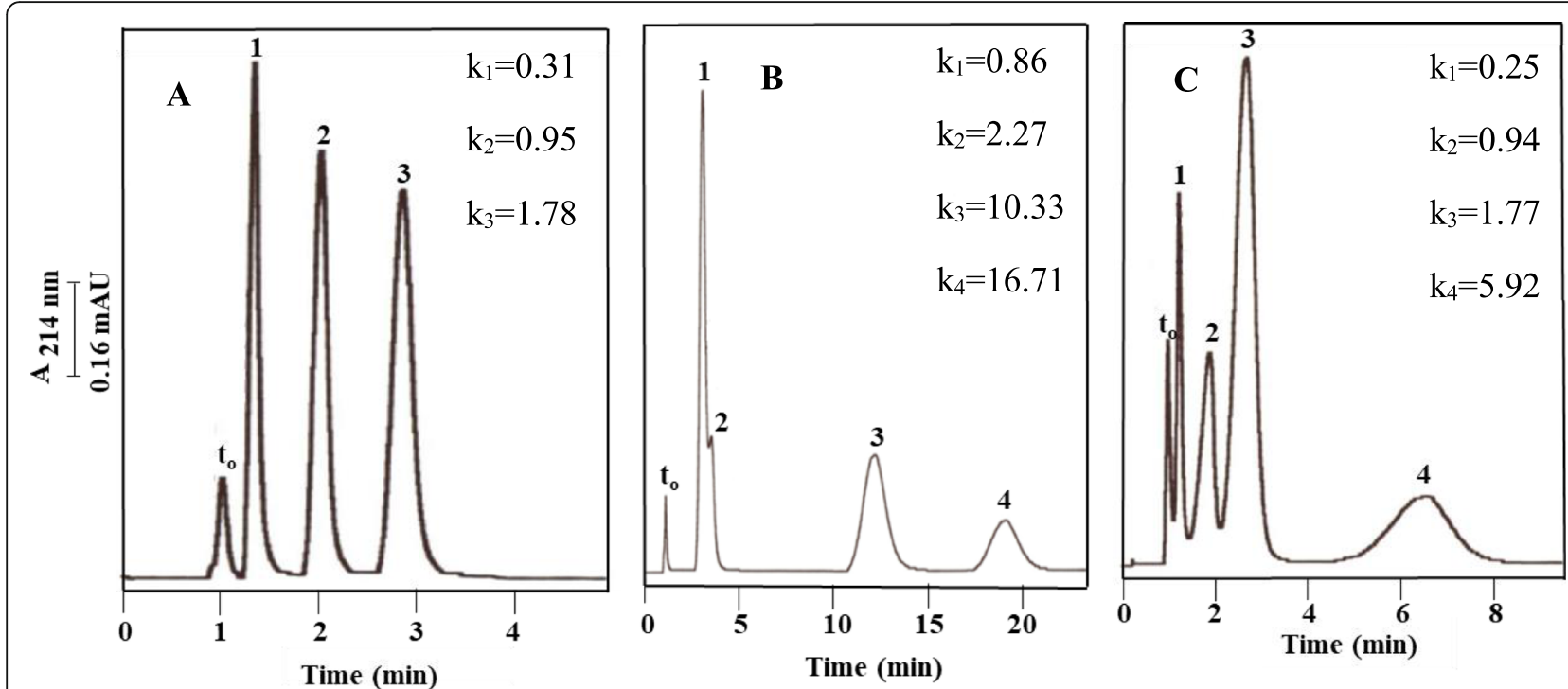

Fig. 7 Chromatograms of small neutral polar solutes (a), nucleobases (b), and organic acids (c) obtained on $0.6 \mathrm{mg}$ of CN-FSNPs/cm-poly(GMMco-EDMA) column. For $\mathbf{a}, \mathbf{b}$, and $\mathbf{c}$, the conditions and the order of solute elution are the same as in Figs. 2a, b, and c, respectively

was directly proportional to the incorporated amount of CN-FSNPs in the column except for the one which contained the highest tested amount of CN-FSNPs (i.e., $4.73 \mathrm{mg}$ of CN-FSNPs per $\mathrm{cm}$ column length) in the study. This deviation of the column backpressure might have resulted from the imperfectly formed monolithic structure due to the highest content of CN-FSNPs in it.

The chromatographic behaviors of CN-FSNPspoly(GMM-co-EDMA) monolithic columns were further evaluated using a wide range of solutes. Using the same mobile phase compositions which were used in evaluating the bare FSNPs-poly(GMM-co-EDMA) monolithic column, the tested small neutral polar solutes, nucleobases, organic acids, food additives, and vitamins eluted from the CN-FSNPs-poly(GMM-coEDMA) monolithic column following the same orders which were obtained on the bare FSNP-entrapped monolithic column, with the exception that $( \pm)$-propranolol eluted first, followed by $( \pm)$-ephedrine and then $( \pm)$-phenylpropanolamine. This observed change

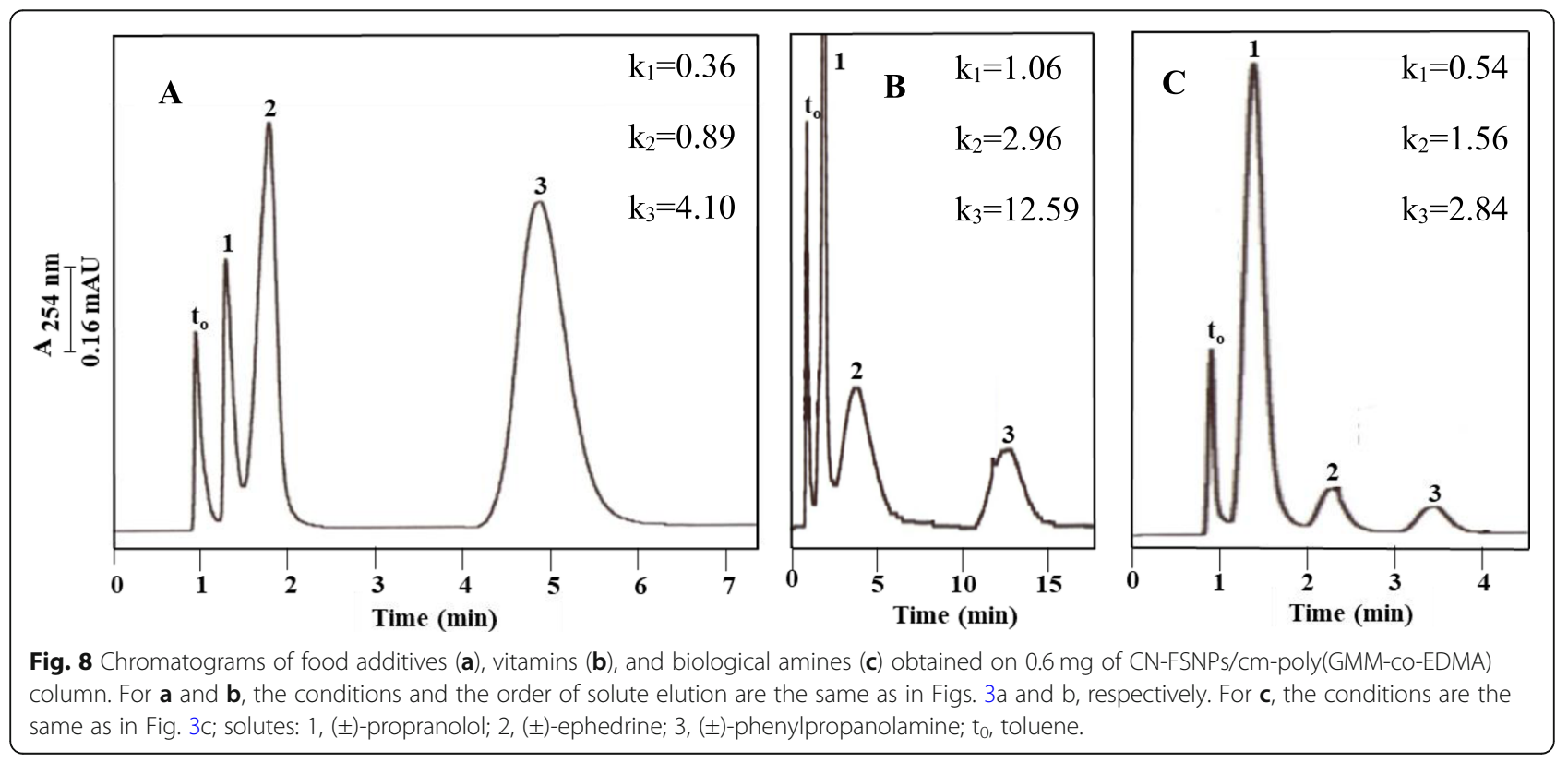


in the elution order may have resulted from the complicated HILIC interactions of the solutes with the attached polar organosilane on the FSNP surface. Furthermore, the highest $k$ values in the separation of small neutral polar solutes, nucleobases, organic acids, food additives, and vitamins were resulted in the column that contained $2.3 \mathrm{mg}$ of CN-FSNPs per cm column length. The column which contained $1.2 \mathrm{mg}$ of CN-FSNPs per $\mathrm{cm}$ column length yielded the highest $k$ values for the separation of tested biological amines.

As has been mentioned earlier, the column which contained $0.6 \mathrm{mg}$ of CN-FSNPs $/ \mathrm{cm}$ was chosen as the best column with the optimum content of the nano-entities since it yielded the highest average separation efficiency for the tested small, neutral, polar solutes (see Fig. 6). The separations of polar and neutral solutes, nucleobases, organic acids, food additives, vitamins, and biological amines obtained on this column with $0.6 \mathrm{mg}$ of $\mathrm{CN}-\mathrm{FSNPs} / \mathrm{cm}$ are shown in Figs. $7 \mathrm{a}-\mathrm{c}$ and $8 \mathrm{a}-\mathrm{c}$, respectively. The $k$ values and the $\alpha$ values obtained on the column are included in Table 1. Also, it should be noted that the $\alpha$ values were noticeably high which led to a good separation of the solutes.

Reproducibility The reproducibility of the analysis was investigated using DMF, formamide, and thiourea as the model solutes. This was carried out by measuring the $k$ values of the just mentioned analytes on the column which contained $0.6 \mathrm{mg}$ of CN-FSNPs per $\mathrm{cm}$ column length, from run-to-run $(n=3)$, day-to-day $(n=3)$, and column-to-column $(n=2)$ using a mobile phase at $95 \%$ $(\mathrm{v} / \mathrm{v}) \mathrm{ACN}$ and $5 \%(\mathrm{v} / \mathrm{v})$ water. The day-to-day and column-to-column reproducibilities were calculated by averaging triplicate measurements of the $k$ values on each day and on each column. In all cases, the reproducibility was determined by the \%RSD of the $k$ values. The average $\%$ RSDs were $2.49 \%, 2.60 \%$, and $2.47 \%$ for run-to-run, day-to-day, and column-to-column, respectively. Moreover, the run-to-run and day-to-day reproducibilities of the other prepared columns (i.e., which contained 1.2, 2.3, and $4.7 \mathrm{mg}$ of CN-FSNPs/cm) were also measured in the same way in order to check the possible bleeding of the nanoparticles from the monolith. For all the above tested columns, the average \%RSDs was $<5 \%$ for run-to-run analyses. However, the \%RSDs of the $k$ values which were obtained on the 4.7 CN-FSNPs $\mathrm{mg} / \mathrm{cm}$ column were significantly increased to $10.6 \%$ for day-to-day analyses. This result further confirms the adverse effects of entrapping high amount of CN-FSNPs into the poly(GMM-co-EDMA) monolith. However, $<5 \%$ RSDs were observed for the $k$ values obtained for the rest of the columns (i.e., 1.2 and $2.3 \mathrm{CN}$ FSNPs $\mathrm{mg} / \mathrm{cm}$ ) in evaluating the day-to-day reproducibilities.
Table $1 \mathrm{k}$ and a values obtained on $0.6 \mathrm{mg}$ CN-FSNPs/cmpoly(GMM-co-EDMA) monolithic column

\begin{tabular}{|c|c|c|}
\hline Analyte & Retention factor, $k$ & Selectivity factor, a \\
\hline DMF & $0.31( \pm 0.01)$ & \\
\hline
\end{tabular}

Formamide $\quad 0.95( \pm 0.04)$

Thiourea $\quad 1.78( \pm 0.03)$

Thymine $\quad 1.86( \pm 0.01)$

1.2

Uracil $\quad 2.27( \pm 0.03)$

Adenine $\quad 10.33( \pm 0.26)$

Cytosine $\quad 16.71( \pm 0.50)$

m-Toluic acid $\quad 0.25( \pm 0.01)$

Salicylic acid $\quad 0.94( \pm 0.01)$

Sulfanilic acid $\quad 1.77( \pm 0.03)$

Sulfosalicylic acid

$5.92( \pm 0.21)$

Benzoic acid

$0.36( \pm 0.01)$

Maleic acid

$0.89( \pm 0.03)$

Ascorbic acid

$4.10( \pm 0.02)$

Nicotinamide

$1.06( \pm 0.04)$

Riboflavin

$2.96( \pm 0.14)$

Thiamine $\mathrm{HCl}$

$12.59( \pm 0.24)$

(士)-Propranolol $\mathrm{HCl}$

$0.54( \pm 0.00)$

( \pm )-Ephedrine $\mathrm{HCl}$

$1.56( \pm 0.01)$

( \pm )-Phenylpropanolamine $\mathrm{HCl} \quad 2.84( \pm 0.02)$

All the analyses were carried out under following common conditions; column, $10 \mathrm{~cm} \times 4.6 \mathrm{~mm}$ i.d; flow rate, $1 \mathrm{~mL} / \mathrm{min}$; injection volume, $20 \mu \mathrm{L}$; temperature, room temperature. Other conditions for DMF, formamide, and thiourea: mobile phase, $95 \%$ ACN $(v / v), 5 \%$ water $(v / v)$; $t_{0}$, toluene. For thymine, uracil, adenine, cytosine, $m$-toluic acid, salicylic acid, sulfanilic acid, and sulfosalicylic acid: mobile phase, $95 \% \mathrm{ACN}(\mathrm{v} / \mathrm{v}), 5 \%$ of $10 \mathrm{mM}$ ammonium acetate, $\mathrm{pH} 4.0$ $(\mathrm{v} / \mathrm{v}) ; \mathrm{t}_{0}$, toluene. For benzoic acid, maleic acid, and ascorbic acid: mobile phase, $90 \%$ ACN $(v / v), 10 \%$ of $10 \mathrm{mM}$ ammonium acetate, $\mathrm{pH} 3.0(\mathrm{v} / \mathrm{v}) ; \mathrm{t}_{0}$ phenyl heptane. For nicotinamide, riboflavin, and thiamine: mobile phase, $90 \%$ ACN $(\mathrm{v} / \mathrm{v}), 10 \%$ of $10 \mathrm{mM}$ ammonium acetate, $\mathrm{pH} 3.0(\mathrm{v} / \mathrm{v}) ; \mathrm{t}_{0}$, toluene. For $( \pm)$-phenylpropanolamine, $( \pm)$-propranolol, and ( \pm )-ephedrine: mobile phase, $95 \%$ ACN $(v / v), 5 \%$ of $200 \mathrm{mM}$ ammonium acetate, $\mathrm{pH} 5.0(\mathrm{v} / \mathrm{v}) ; \mathrm{t}_{0}$, phenyl heptane 


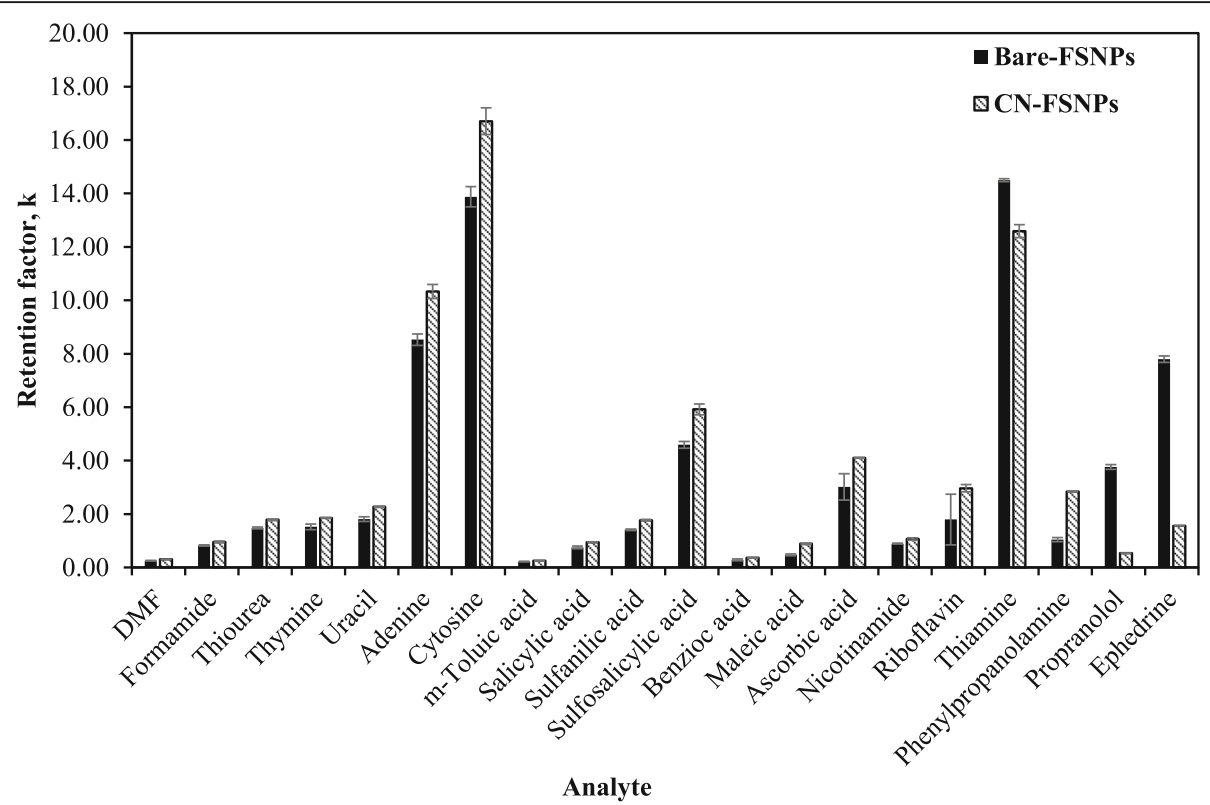

Fig. 9 Comparison of the $k$ values obtained on FSNPs-poly(GMM-co-EDMA) column to those obtained on the CN-FSNPs-poly(GMM-Co-EDMA) column under the same experimental conditions

Comparison of the retentive properties of the monolithic columns incorporated with bare and cyano-modified FSNPs As shown in Fig. 9, the solute retention in terms of $k$ values obtained on the $\mathrm{CN}$ FSNPs-poly(GMM-co-EDMA) monolithic column were compared to those obtained on the bare FSNPspoly(GMM-co-EDMA) monolithic column. As can be seen in this figure, all the tested analytes showed an increased retention on the CN-FSNPs-poly(GMM-coEDMA) monolithic column, except thiamine, propranolol, and ephedrine, compared to that of the FSNPspoly(GMM-co-EDMA) monolithic column. This observation is attributed to the increased polarity and in turn the increased hydrophilic interactions between the attached polar organosilanes in cyano-modified FSNPs and the test solutes. On the other hand, higher $k$ values were obtained for the basic solutes, e.g., thiamine, propranolol, and ephedrine, on the bare FSNPs-poly(GMMco-EDMA) monolith, which can be attributed to the silanophilic interactions of bare silica with the basic solutes, which involve electrostatic interactions of the negatively charged surface silanols of the bare FSNPs with the positively charged amino groups of the solutes.

\section{Conclusions}

Poly(GMM-co-EDMA) monolith served as an ideal matrix for the incorporation of both bare FSNPs and surface-modified FSNPs with polar $\mathrm{CN}$-functionalities. The prepared bare FSNPs- poly(GMM-co-EDMA) and CN-FSNPs-poly(GMM-co-EDMA) monoliths were successfully used in the HILIC separations of a wide range of analytes including neutral polar and low molecular weight small solutes, nucleobases, nucleosides, organic acids, food additives, vitamins, and biological amines. The separations yielded high selectivity factors, which led to full separations between the solutes. Considerably increased retention of the analytes was achieved due to the modification of the silica surface with the polar organosilane, 2-cyanoethyltrichlorosilane compared to the unmodified FSNPs.

\section{Abbreviations}

ACN: Acetonitrile; AIBN: 2,2'-Azobis(isobutyronitrile); EDMA: Ethylene glycol dimethacrylate; FSNPs: Fumed silica nanoparticles; GMM: Glyceryl monomethacrylate; CN-FSNPs: Cyano-modified fumed silica nanoparticles

\section{Acknowledgements}

Not applicable.

\section{Authors' contributions}

NG carried out the experimental studies, analysis, data interpretation, and wrote the manuscript. ZER supervised the entire work and edited the manuscript. All authors read and approved the final manuscript.

\section{Funding}

Not applicable.

Availability of data and materials

Research data have been provided in the manuscript.

Competing interests

The authors declare that they have no competing interests. 
Received: 20 May 2020 Accepted: 20 August 2020

Published online: 04 September 2020

\section{References}

AEROSIL(R) Fumed silica - technical overview. Available via https://www.aerosil. com/sites/lists/RE/DocumentsSI/Technical-Overview-AEROSIL-Fumed-SilicaEN.pdf. (n.d.) Accessed 25 May 2017

Alpert AJ. Hydrophilic-interaction chromatography for the separation of peptides, nucleic acids and other polar compounds. J Chromatogr A. 1990;499:177-96.

Aydoğan C. Boronic acid-fumed silica nanoparticles incorporated large surface area monoliths for protein separation by nano-liquid chromatography. Anal Bioanal Chem. 2016;408(29):8457-66.

Aydogan C, El Rassi Z. Monolithic stationary phases with incorporated fumed silica nanoparticles. Part I. Polymethacrylate-based monolithic column with incorporated bare fumed silica nanoparticles for hydrophilic interaction liquid chromatography. J Chromatogr A. 2016a;1445:55-61. https://doi.org/ 10.1016/j.chroma.2016.03.075.

Aydogan C, El Rassi Z. Monolithic stationary phases with incorporated fumed silica nanoparticles. Part II. Polymethacrylate-based monolithic column with "covalently" incorporated modified octadecyl fumed silica nanoparticles for reversed-phase chromatography. J Chromatogr A. 2016b;1445:62-7. https:// doi.org/10.1016/j.chroma.2016.03.083.

Buszewski B, Noga S. Hydrophilic interaction liquid chromatography (HILIC) -a powerful separation technique. Anal Bioanal Chem. 2012;402(1):231-47.

Combs Jr, G. F., \& McClung, J. P. (2016). The vitamins: fundamental aspects in nutrition and health: Academic press.

Courtois J, Byström E, Irgum K. Novel monolithic materials using poly (ethylene glycol) as porogen for protein separation. Polymer. 2006;47(8):2603-11.

Gama MR, da Costa Silva RG, Collins CH, Bottoli CB. Hydrophilic interaction chromatography. TrAC Trends Anal Chem. 2012;37:48-60.

Gong Z-S, Duan L-P, Tang A-N. Amino-functionalized silica nanoparticles for improved enantiomeric separation in capillary electrophoresis using carboxymethyl- $\beta$-cyclodextrin (CM- $\beta-C D)$ as a chiral selector. Microchimica Acta. 2015;182(7-8):1297-304.

Guiochon G. Monolithic columns in high-performance liquid chromatography. J Chromatogr A. 2007;1168(1):101-68.

Gunasena DN, El Rassi Z. Neutral, charged and stratified polar monoliths for hydrophilic interaction capillary electrochromatography. J Chromatogr A. 2013;1317:77-84

Holdšvendová P, Suchánková J, Bunček M, Bačkovská V, Coufal P. Hydroxymethyl methacrylate-based monolithic columns designed for separation of oligonucleotides in hydrophilic-interaction capillary liquid chromatography. J Biochem Biophys Methods. 2007;70(1):23-9.

Jandera P. Stationary and mobile phases in hydrophilic interaction chromatography: a review. Anal Chim Acta. 2011;692(1):1-25.

Jaoudé MA, Lassalle Y, Randon J. Separation of xanthines in hydro-organic and polar-organic elution modes on a titania stationary phase. J Sep Sci. 2014; 37(5):536-42.

Jiang Z, Smith NW, Liu Z. Preparation and application of hydrophilic monolithic columns. J Chromatogr A. 2011;1218(17):2350-61.

Karatapanis AE, Fiamegos YC, Stalikas CD. Study of the behavior of water-soluble vitamins in HILIC on a diol column. Chromatographia. 2010;71(9-10):751-9.

Kučera $R$, Kovaříková $P$, Klivický $M$, Klimeš J. The retention behaviour of polar compounds on zirconia based stationary phases under hydrophilic interaction liquid chromatography conditions. J Chromatogr A. 2011; 1218(39):6981-6.

LV Y, Lin Z, Svec F. "Thiol-ene" click chemistry: a facile and versatile route for the functionalization of porous polymer monoliths. Analyst. 2012;137(18):4114-8.

Mayadunne E, El Rassi Z. Facile preparation of octadecyl monoliths with incorporated carbon nanotubes and neutral monoliths with coated carbon nanotubes stationary phases for HPLC of small and large molecules by hydrophobic and $\pi-\pi$ interactions. Talanta. 2014;129:565-74. https://doi.org/ 10.1016/j.talanta.2014.06.032

Melander W, Horvath C, Horvath C. High performance liquid chromatographyadvances and perspectives, vol. 2. New York: Academic Press; 1980. p. 113319.

Svec F. Preparation and HPLC applications of rigid macroporous organic polymer monoliths. J Sep Sci. 2004;27(10-11):747-66.

Tong S, Zhou X, Zhou C, Li Y, Li W, Zhou W, Jia Q. A strategy to decorate porous polymer monoliths with graphene oxide and graphene nanosheets. Analyst. 2013;138(5):1549-57.
Wang Y, Baeyens WR, Huang C, Fei G, He L, Ouyang J. Enhanced separation of seven quinolones by capillary electrophoresis with silica nanoparticles as additive. Talanta. 2009;77(5):1667-74.

Xu S, Mo R, Jin C, Cui X, Bai R, Ji Y. Mesoporous silica nanoparticles incorporated hybrid monolithic stationary phase immobilized with pepsin for enantioseparation by capillary electrochromatography. J Pharm Biomed Anal. 2017;140:190-8.

Xu Y, Cao Q, Svec F, Frechet JMJ. Porous polymer monolithic column with surface-bound gold nanoparticles for the capture and separation of cysteinecontaining peptides. Anal Chem (Washington, DC, U S). 2010;82(8):3352-8. https://doi.org/10.1021/ac1002646.

\section{Publisher's Note}

Springer Nature remains neutral with regard to jurisdictional claims in published maps and institutional affiliations.

\section{Submit your manuscript to a SpringerOpen ${ }^{\circ}$ journal and benefit from:}

- Convenient online submission

- Rigorous peer review

- Open access: articles freely available online

- High visibility within the field

- Retaining the copyright to your article

Submit your next manuscript at $\boldsymbol{\nabla}$ springeropen.com 